\title{
Effect of dietary restrictions on disease activity in rheumatoid arthritis
}

\author{
DEEPA BERI, A N MALAVIYA, RENU SHANDILYA, AND R R SINGH \\ From the Clinical Immunology Section, Department of Medicine, All India Institute of Medical Sciences, New \\ Delhi 110029, India
}

SUMMARY Additions in five steps were made, as a possible therapeutic measure, to the diet of 27 patients with rheumatoid arthritis (RA) after a period of two weeks of a basal isocaloric diet free from pulses, cereals, milk, and non-vegetarian protein foods. Fourteen patients finally took part in the trial, $10(71 \%)$ of whom showed significant clinical improvement. Only three patients $(11 \%)$ adhered to the diet for a period of 10 months. The others discontinued the diet and were then treated with conventional disease modifying drugs. The study indicates that dietary factors may influence inflammatory response in RA.

Key phrases: food intolerance in RA, individual susceptibility to food in RA, dietary manipulation in RA, response assessment in RA.

Recently there has been a resurgence of interest in the relation between diet and RA. ${ }^{1-3}$ Some reports have shown definite and objective correlation between certain dietary factors and the inflammation of RA. ${ }^{4-8}$ The present study was undertaken to analyse systematically the effect of various dietary components on the inflammatory activity of this disease.

\section{Patients and methods}

PATIENTS

Twenty seven patients with definite $\mathrm{RA}^{9}$ were included in the study. None of the patients had previously received any disease modifying antirheumatoid drugs.

\section{I E T}

The details of the study were explained to the patients and their informed consent obtained before the study began. Before the start of the dietary elimination therapy, patients who were receiving non-steroidal anti-inflammatory drugs (NSAIDs) began a wash out period of two weeks. During this time patients were allowed a normal diet and $500 \mathrm{mg}$ of paracetamol as required for pain relief.

Accepted for publication 8 June 1987.

Correspondence to $\mathrm{Dr} A \mathrm{~N}$ Malaviya. Clinical Immunology Section, Department of Medicine. All India Institute of Medical Sciences, Ansari Nagar. New Delhi 110029. India.
A complete clinical and laboratory assessment was carried out at the beginning and at the end of the wash out period. Patients not requiring a wash out period were immediately placed on the dietary therapy after the initial assessment.

For the first two weeks of the dietary therapy, patients were allowed an isocaloric diet consisting of fruit, vegetables, sugar, and refined oil (diet I). This was followed by a repeat estimation of the erythrocyte sedimentation rate (ESR) (Westergren) and clinical variables, after which the patient was given diet II, consisting of diet I plus all pulses, for another period of two weeks. The clinical and laboratory assessments were repeated as before. Two further diets, diet IIIA, consisting of diet I plus wheat and wheat products, and diet IIIB, including rice and rice products in addition to diet $\mathrm{I}$, were again given for a period of two weeks each and were each followed by assessments. These diets were followed by stepwise two weekly additions of milk and milk products (diet IV) and non-vegetarian food (diet V), consisting of eggs, meat, fish, and chicken, with regular clinical and laboratory estimations as before.

If any increase in symptoms was observed after a particular diet the patients were returned to diet I until improvement was observed before attempting the next diet. Thus groups of foods causing exacerbation of the disease activity were determined and eliminated from the diet, with long term 
assessment every three months. During the entire period of dietary therapy patients were allowed to take paracetamol tablets if required.

The criteria for clinical assessment included morning stiffness, joint pain score, articular index, and patients' global assessment. Morning stiffness was assessed arbitrarily, i.e., grade $I=15 \mathrm{~min}$, grade II = up to 1 hour, grade III = up to $2^{1 / 2}$ hours, grade IV $=2 \frac{1}{2}$ hours or more. Joint pain score was marked according to the criteria of Deodhar et al, ${ }^{10}$ and the articular index was determined by Ritchie's method. ${ }^{11}$ Patients' global assessment was graded as follows: grade $\mathrm{I}=$ much better, grade $\mathrm{II}=$ better, grade III= unchanged, grade IV=worse, and grade $\mathrm{V}=$ much worse. Initial laboratory estimations included haemoglobin, total leucocyte estimation, and ESR by standard methods. Latex fixation titre (Hoechst (India) Pvt Ltd) was also estimated at the beginning of the trial. The ESR was repeated every two weeks, i.e., at the initiation/completion of each diet step.

A record of the patient's weight was also maintained in order to ensure that no excessive weight loss took place (i.e., $>5 \%$ ).

\section{Results}

In the present study only $14(52 \%)$ of the 27 patients could take the diet as advised, the rest being unable to complete the first step.

Of the 14 patients who carried out the dietary instuctions, $10(71 \%)$ showed significant clinical improvement. The remaining four patients did not show any benefit from the first and second diets and were changed to standard drug therapy.

Of the 10 responsive patients, one responded to elimination of pulses alone, four to the elimination of both the cereals (wheat and rice), and two to the exclusion of rice and rice products. For the remairo ing three patients improvement was observed on on the elimination of multiple food items, i.es

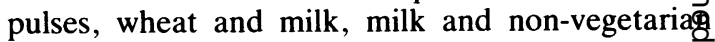
food, and pulses and non-vegetarian food.

The pattern of response for these 10 patients wâs as follows: after diet I these patients showed a mean percentage improvement in the clinical variables ranging from 25 to $54 \%$, as compared with thegp

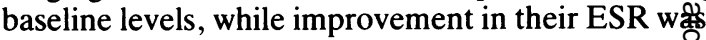
$33 \%$. Introduction of diet II led to deterioration the condition of three patients (Table 1), one of whom showed a rapid flare of symptoms. Thes remaining two patients complained of exacerbation of their disease, particularly with one pulse (Bengæl Gram). The other seven patients showed clinical improvement and a mean percentage improvemeat of $45 \%$ in their ESR. One patient dropped out of the trial at this stage and could not be contacted again.

Diet III (A and B) led to significant deterioratiog in the condition of the remaining six patients, of whom four worsened with both the cereals and two with rice and rice products (Tables 1 and 2). Drog therapy was initiated for the first group as it ws difficult to prevent their consuming cereals entirelo Four patients entered diet IV, of whom two deteriorated and two improved. Two patien $\overrightarrow{\vec{w} \mathrm{~s}}$ reached the last stage, i.e., diet $\mathrm{V}$, and both deteriorated.

Considerable weight loss $(9 \%)$ was observed in five patients, but there was no difference between the weight loss of good and poor responders.

At the time of reporting, three patients (11\%)

Table 1 Variation of the erythrocyte sedimentation rate (ESR) in response to various food groups

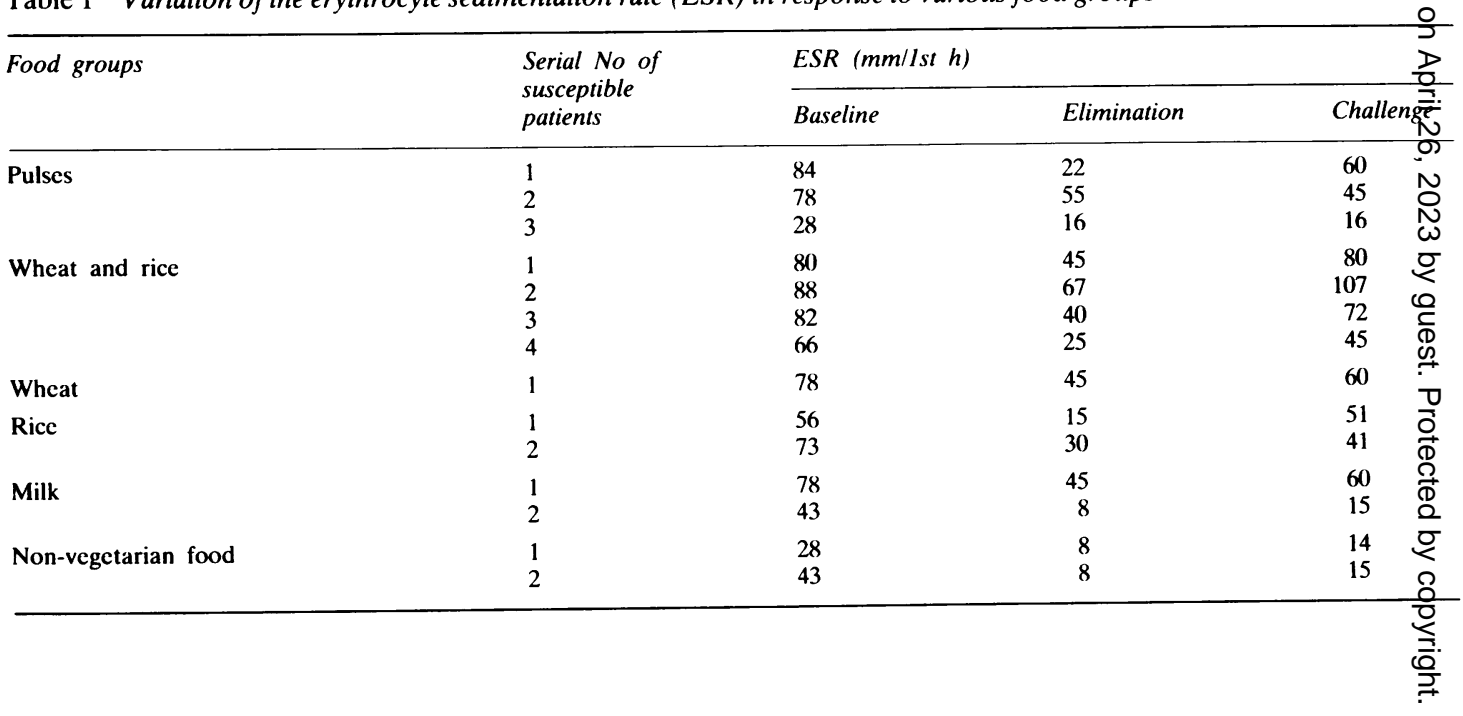


Table 2 Variation of the global assessment in response to various food groups

\begin{tabular}{lllll}
\hline Food groups & $\begin{array}{l}\text { Serial No of } \\
\text { susceptible } \\
\text { patients }\end{array}$ & \multicolumn{2}{l}{ Global assessment } & \\
\cline { 3 - 5 } & 1 & Baseline & Elimination & Challenge \\
\hline Pulses & 2 & 4 & 1 & 4 \\
& 3 & 4 & 1 & 4 \\
Wheat and rice & 1 & 4 & 1 & 3 \\
& 2 & 4 & 1 & 3 \\
Wheat & 3 & 2 & 1 & 4 \\
Rice & 4 & 3 & 1 & 3 \\
Milk & 1 & 4 & 1 & 4 \\
Non-vegetarian food & 1 & 4 & 2 & 2 \\
& 2 & 4 & 1 & 3 \\
& 1 & 4 & 1 & 4 \\
\end{tabular}

were still adhering to dietary restrictions and continue to do well without taking any drugs.

\section{Discussion}

Patients with rheumatoid arthritis frequently complain of an increase in their disease activity on consuming certain food items. ${ }^{12}$ The role of food as a cause of inflammation in rheumatoid arthritis is still controversial, however. A relation between ingested food and fluctuation in disease activity has yet to be determined unequivocally.

Most studies are based on dietary elimination, i.e., a certain food item is eliminated, a challenge is then made with the same dietary item, and observations are recorded. ${ }^{124}$ With this method it is usually difficult to establish multiple food 'allergies' in a given patient. In the present study, therefore, the method consisted of 'diet additions' after a period of wash out, rather than elimination. Although this method was very cumbersome, one of the main advantages was that the responders could be identified immediately. The results showed that a high proportion of patients improved on dietary manipulations, and that there was marked individual variation in response to the elimination of different dietary items. Some patients improved on elimination of only one of the food items, whereas others were susceptible to more than one dietary component. The study also highlighted the practical difficulty involved in instituting dietary treatment as a major mode of treatment in RA as the follow up was difficult and the drop out rate was high. The present work indicates, however, that individual susceptibility (allergy?, unusual immune response?) may be an important area for future investigations of the pathogenesis of RA.

One possible mode of action of dietary therapy in RA has been considered to be non-specific suppression of inflammation by suppression of synthesis of proinflammatory prostaglandins. ${ }^{12}$ An alternative possibility could be abnormal handling of the food antigens by the host. A possible future line of work may be to identify the food allergies (intolerance?) in patients with RA and to try to correlate their humoral and cell mediated immune response against these food antigens in the blood and joint fluid.

As mentioned earlier the dietary addition procedure used in this study was complicated and cumbersome, yet, in contrast with the usual elimination-challenge methods had the advantage that the responders could be identified immediately. Possibly an easier approach would be to give a basal isocaloric diet free from all the major food groups followed by a rotation diet with elimination alternating with challenge at shorter intervals. Such a study is in progress.

This work was supported by a 'grant-in-aid' from ICMR, New Delhi, India.

\section{References}

1 Panush R S, Carter R L, Katz P, Kowsari B, Longley S, Finnie S. Diet therapy for rheumatoid arthritis. Arthritis Rheum 1983; 26: $462-71$.

2 Panush R S, Stround R M, Webster E M. Food-induced (allergic) arthritis. Arthritis Rheum 1986; 29: 220-6. 
3 Ziff M. Diet in the treatment of rheumatoid arthritis. Arthritis Rheum 1983; 26: 457-61.

4 Darlington L G, Ramsey N W, Mansfield J R. Placebocontrolled, blind study of dietary manipulation therapy in rheumatoid arthritis. Lancet 1986; i: 236-8.

5 Sköldstam L, Larson L, Lindström F D. Effect of fasting and lactovegetarian diet on rheumatoid arthritis. Scand J Rheumatol 1979; 8: 249-56.

6 Lucus C P, Power L. Dietary fat aggravates active rheumatoid arthritis [Abstract]. Clin Res 1981; 29: 754A.

7 Parke A L, Hughes G R V. Rheumatoid arthritis and food: a case study. $\mathrm{Br}$ Med $J$ 1981; 282: 2027-9.

8 Sundqvist T, Lindström F, Magnusson K E, Sköldstam L, Stjernström I, Tagesson C. Influence of fasting on intestinal permeability and disease activity in patients with rheumatoid arthritis. Scand J Rheumatol 1982; 11: 33-8.

9 Ropes M W, Bennett G A, Cobb S, Jacox R, Jessar R A Revision of diagnostic criteria for rheumatoid arthritis. But Rheum Dis 1958; 9: 175-6.

10 Deodhar S D, Dick W C, Hodgkinson R, Buchanan W W Measurement of clinical response to anti-inflammatory drugD therapy in rheumatoid arthritis. $Q J$ Med 1973; 42: 387-401.

11 Ritchie D M. Clinical studies with articular index for thछे assessment of joint tenderness in patients with rheumatoid arthritis. $Q J$ Med 1968; 37: 393-406.

12 Kremer J M, Michalek A V, Lininger L, et al. Effects of manipulation of dietary fatty acids on clinical manifestations ow rheumatoid arthritis. Lancet 1985 ; i: 184-7. 